\title{
Method to solve integral equations of the first kind with an approximate input
}

\author{
Victor D. Efros* \\ European Centre for Theoretical Studies in Nuclear Physics and Related Areas (ECT*), \\ Villa Tambosi, I-38123 Villazzano (Trento), Italy ${ }^{\dagger}$
}

\begin{abstract}
Techniques are proposed for solving integral equations of the first kind with an input known not precisely. The requirement that the solution sought for includes a given number of maxima and minima is imposed. It is shown that when the deviation of the approximate input from the true one is sufficiently small and some additional conditions are fulfilled the method leads to an approximate solution that is necessarily close to the true solution. No regularization is required in the present approach. Requirements on features of the solution at integration limits are also imposed. The problem is treated with the help of an ansatz proposed for the derivative of the solution. The ansatz is the most general one compatible with the above mentioned requirements. The techniques are tested with exactly solvable examples. Inversions of the Lorentz, Stieltjes and Laplace integral transforms are performed, and very satisfactory results are obtained. The method is useful, in particular, for the calculation of quantum-mechanical reaction amplitudes and inclusive spectra of perturbation-induced reactions in the framework of the integral transform approach.

PACS numbers: 95.75.Pq, 02.30.Zz, 02.90.+p, 03.65.Nk, 24.10.Cn
\end{abstract}

\footnotetext{
*v.efros@mererand.com

$\dagger$ on leave from National Research Centre "Kurchatov Institute", 123182 Moscow, Russia
} 


\section{INTRODUCTION}

Inversion of integral transforms which values are known only approximately is a longstanding issue. The situation is unfavorable when the solution that corresponds to an approximate input differs from the true solution in a way that it includes extra narrow peaks or quick oscillations. In these cases small changes in the input correspond to changes in the solution that are not small.

But in such cases the numbers of maxima or minima of the approximate solution and of the true solution would differ from each other. Therefore, to cure the situation it is suggested in the present paper to seek for the approximate solution in the class of functions having the same number of maxima and minima as the true solution has. In general this number is not known but often it is not hard to guess it as discussed below. A general ansatz for the solution compatible with a prescribed number of maxima and minima is proposed below for performing inversion.

In Sec. 2 the proposed inversion method is described. It is applicable to a wide class of integral equations of the first kind.

In particular, the techniques are aimed to increase ability of the integral transform approach used to calculate amplitudes and inclusive spectra of perturbation-induced reactions in the framework of nuclear physics. An outline of that approach is presented in Sec. 3.

In Sec. 4 the techniques are tested with exactly solvable examples. The Laplace, Stieltjes and so called Lorentz [1] integral transforms are inverted.

In Sec. 5 the issue of convergence of the method to the true solution is investigated. The important point is that the solution is sought for in a restricted class of functions. This ensures the convergence. (A different case with such features exists, and in this case the solution belongs to a known compactum, see e.g. [2].)

\section{INVERSION METHOD}

The integral equation

$$
\int_{E_{t h r}}^{\infty} K(\sigma, E) f(E) d E=\Phi(\sigma), \quad \sigma_{1} \leq \sigma \leq \sigma_{2}
$$

is studied. All the quantities are real, the solution $f$ is smooth and unique. The $K$ operator is assumed to be continuous at the norm definitions specified below. The exact $\Phi$ is not 
known so that one is forced to deal with an approximation to $\Phi$ denoted below $\Phi_{a p p r}$. In the case of few-body calculations performed in the framework of the approach outlined in Sec. 3 the accuracy of an input $\Phi_{a p p r}$ is normally at a per cent level. (The notation for the lower integration limit in Eq. (1) is used in connection with the problems addressed in Sec. 3 and 4 . In the same connection the upper integration limit is set to be infinite.)

As it is known the solution $f$ sought for may be unstable with respect to small changes of the input. In the literature, various regularization procedures were put forward to suppress the instability. (A rather complete review can be found in Refs. [2, 3], see also e.g. [4, 5].) In particular, within the approach outlined in Sec. 3 the following regularization procedure was always used so far. The solution was approximated by the expression

$$
f_{N}(E)=\sum_{n=1}^{N} c_{n} \varphi_{n}(E, \alpha)
$$

where $\left\{\varphi_{n}\right\}$ is a set of basis functions. The linear parameters $c_{n}$ and the non-linear parameter $\alpha$ were found via fitting the quantity $K f_{N}$ to $\Phi_{\text {appr }}$. The regularization was realized with the choice of the $N$ value. This value should be not too high to exclude unstable behavior. At the same time it should be not too low so that the approximation of the true $f(E)$ with the Eq. (2) type expression would be sufficiently accurate. (See e.g. Ref. [6] for further details.) The problem is that the two conditions are compatible with each other only to a certain degree. If the accuracy in an input $\Phi$ is not high enough reasonable inversion may become impossible or tricky. The issue of the proper choice of the regularization parameter does not arise in the method of the present paper since no regularization is required here.

The techniques proposed below are designed to increase the accuracy of inversion at a given accuracy in $\Phi$. It should be noted in this connection that when Eq. (2) is used another source of instability exists besides uncertainties in the input. This instability is related to arising ill-conditioned systems of linear equations for the expansion coefficients from Eq. (2). The influence of corresponding round-off errors on the solution becomes considerable when $N$ increases even if the exact input is employed. In the framework of the method described below the round-off errors are probably less important.

It is shown in the last section that when small uncertainties in an input are of nonrandom nature and the round-off errors in calculation are not substantial no other sizable deviations from the true solution besides narrow peaks of small strength may exist in the problem. Adopting that this is the case let us impose the requirement that the solution 
sought for includes a given number of maxima and minima. Then one may conclude, see also the last section, that if the guess as to their number is correct then those narrow peaks are excluded and correspondingly the approximate solution is close to the exact solution "almost everywhere".

The only possible exception are the points of maxima and minima of the solution where extra narrow peaks might appear. If this improbable situation takes place these peaks are in general to be simply removed. Indeed, true narrow peaks may appear only when there exist specific physical reasons for this, such as resonant states. When the above requirement on the number of maxima and minima is imposed no regularization is required. Below the solution is sought for in the class of functions satisfying this requirement.

In many cases incorrect guesses as to the number of maxima and minima of the solution are easily rejected. Some maxima or minima may be very weakly pronounced. In such a case it may occur that approximate solutions with different numbers of maxima and minima are very close to each other and lead to the fits of a similar quality. Each of these approximate solutions is acceptable. Apart from this case, if the inversion is performed under the condition that the number of maxima and minima is less than the true one this necessarily should lead to a fit of a lower quality. And if the inversion is performed under the condition that their number is higher than the true one this should lead either to a fit of lower quality or to unrealistically narrow extra peaks in the solution with peaks positions and amplitudes being unstable.

The inversion procedure is as follows. In the problems addressed in Sec. 3 the threshold behavior of $f(E)$ at $E \rightarrow E_{t h r}$ is known. ${ }^{1}$ In particular, one has $f\left(E_{t h r}\right)=0$. Also $f(\infty)=0$. Let us rewrite Eq. (1) in terms of $d f / d E \equiv f^{\prime}(E)$ integrating by part:

$$
\int_{E_{t h r}}^{\infty} \tilde{K}(\sigma, E) f^{\prime}(E) d E=\Phi(\sigma)
$$

$\left(\tilde{K}(\sigma, E)=-\int K(\sigma, E) d E\right.$.) Eq. (3) supplemented with the conditions

$$
f\left(E_{t h r}\right)=0, \quad f(\infty)=0
$$

is equivalent to Eq. (1) at inputs $\Phi$ that lead to the values (4). To satisfy the conditions (4)

\footnotetext{
${ }^{1}$ This behavior is deduced from the known behavior at $E_{\gamma} \rightarrow E_{t h r}$ of the matrix elements entering Eq. 17). In this limit they include the $E_{\gamma}$ dependence as a factor.
} 
let us impose the requirement

$$
\int_{E_{t h r}}^{\infty} f^{\prime}(E) d E \equiv f(\infty)-f\left(E_{t h r}\right)=0
$$

and subsequently find the solution to Eq. (1) e.g. as $f(E)=\int_{E_{t h r}}^{E} f^{\prime}\left(E^{\prime}\right) d E^{\prime}$. The solution to Eq. (3) with the condition (5) is unique. Indeed, otherwise there would exist more than one solution to Eq. (1) constructed in the above way.

To be specific let us consider below the most frequent case when at high $E$ values the solution $f(E)$ decreases as power of $E$. (In the case of problems addressed in Sec. 3, one can see that in order to lead not to a power but to an exponential decrease of $f(E)$ the inter-particle interaction in the coordinate or momentum representation should be analytic in all the coordinates, or momenta, respectively. This is not the usual case.) Let $f(E)$ have precisely $N$ maxima and minima. Let $\zeta_{t h r}(E)$ be a monotonous factor reproducing the threshold behavior of $f(E)$ at $E \rightarrow E_{t h r}$, behaving as power of $E$ (e.g. as a constant) at $E \rightarrow \infty$, and arbitrary otherwise. The following ansatz for $f^{\prime}(E)$ is suggested,

$$
f^{\prime}(E)=C \zeta_{t h r}^{\prime}(E)\left[\prod_{i=1}^{N}\left(E-E_{i}\right)\right] \frac{e^{\gamma(E)}}{[(E / \bar{E})+1]^{\beta}} .
$$

Here $\zeta_{t h r}^{\prime}(E) \equiv d \zeta_{t h r} / d E, \bar{E}$ and $\beta$ are parameters, $\gamma(E)$ is a smooth function finite both at $E=E_{t h r}$ and at $E \rightarrow \infty$, and e.g. $\gamma\left(E_{t h r}\right)=0$. The parameter $C$ determines the overall normalization. This expression is the most general one for the derivative of a function that has precisely $N$ maxima and minima, a given threshold behavior, and a power decrease at $E \rightarrow \infty$. (Inflection points do not require a special consideration. A given power decrease is provided by the choice of the parameter $\beta$.) The function $\gamma(E)$ may be taken e.g. in the form

$$
\gamma(E)=\frac{\Delta E / \bar{E}}{(\Delta E / \bar{E})+1} \sum_{k=0}^{\infty} \frac{c_{k}}{[(\Delta E / \bar{E})+1]^{k}}
$$

with $\Delta E=E-E_{t h r}$. Eq. (7) allows the description of next-to-leading terms in $f^{\prime}(E)$ when both $E \rightarrow E_{t h r}$ and $E \rightarrow \infty$. The fitting parameters are then $C,\left\{E_{i}\right\}, \bar{E}, \beta$, and $\left\{c_{k}\right\}$.

To satisfy the requirement (5) it is convenient to express one of the $E_{i}$ values in (6) in terms of the other fitting parameters. In certain cases also the sum-rule condition

$$
-\int_{E_{t h r}}^{\infty} E f^{\prime}(E) d E \equiv \int_{E_{t h r}}^{\infty} f(E) d E=S
$$

may be imposed where the value of $S$ is known, see Sec. 3. From this condition it is convenient to express $C$ in terms of the other fitting parameters. 
The remaining parameters are to be determined from the least-square fit procedure

$$
\left\|\Phi_{a p p r}-\tilde{K} f_{M}^{\prime}\right\| \equiv\left\|\Phi_{a p p r}-K f_{M}\right\|=\min
$$

Here $f_{M}^{\prime}$ is an approximation to $f^{\prime}$ of the form (6) with $M$ parameters retained, $f_{M}$ is the corresponding approximation to $f$, and the norm is defined as

$$
\|F\|=\left[\int_{\sigma_{1}}^{\sigma_{2}} w(\sigma) F^{2}(\sigma) d \sigma\right]^{1 / 2}, \quad w(\sigma) \geq 0 .
$$

(When the equation of Eq. (1) type is considered with $a$ and $b$ being the lower and the upper integration limits and when $f(a) \neq 0$ and/or $f(b) \neq 0$ one may proceed in a similar way. One may rewrite the equation in the form of the equation for the derivative $f^{\prime}(E)$ and from that equation one obtains $f^{\prime}(E)=p(E) f(a)+q(E) f(b)+r(E)$ where $p$, $q$, and $r$ are known functions. Then it is easy to get the relation $f(E)=A(E)+\lambda B(E)$ where $\lambda$ may be chosen to be $f(a), f(b)$, or $f(c), c$ being an arbitrary point, and $A$ and $B$ are the corresponding known functions. If there is no point $c$ at which $f(c)$ is known then the parameter $\lambda$ is to be determined from the initial integral equation.)

In simple cases the main features of the solution $f(E)$ are determined by its threshold behavior, its decrease at large $E$, and by positions and amplitudes of its maxima and minima. For example, let us consider here the one-maximum or one-minimum case. The mentioned features of the solution may be reproduced in the framework of the ansatz (6) without the factor $\exp [\gamma(E)]$. Therefore one may think that the solution is rather insensitive to the values of the parameters determining $\gamma(E)$. In addition, in many cases the dependence of the quantity $\left\|\Phi_{a p p r}-\tilde{K} f_{M}^{\prime}\right\|$ to be minimized on the parameters is rather smooth. Then a good strategy to find the global minimum of Eq. (9) would be in the first stage to disregard the factor $\exp [\gamma(E)]$ in Eq. (6) and to seek for the minimum with respect to other parameters on a grid that includes sufficiently large number of points. Grids where one grid is put inside another one may be used. The values of the parameters thus obtained may be used as starting values at a subsequent conventional minimization.

To carry out the latter minimization a good choice are special codes for the least-square fit with non-linear parameters (e.g. Ref. [7], Sec 15.5). Codes that use derivatives are preferable since the derivatives of e.g. the expressions (6) and (7) with respect to fitting parameters are readily calculated. 
At calculating the norm in (9) one replaces the integral with an integral sum. A good approximation to the solution may be obtained even if the number of terms in this sum is insufficient to reproduce the integral.

Practical criteria of the quality of an approximate solution $f_{M}$ thus obtained are both its stability with respect to increase of the number $M$ of fitting parameters at a given $\Phi_{a p p r}$ and its stability with respect to variations of $\Phi_{a p p r}$. If a realistic estimate of $\left\|\Phi-\Phi_{a p p r}\right\|$ is available, say $\left\|\Phi-\Phi_{\text {appr }}\right\|>\epsilon_{0}$, then the criterion of stability with respect to increase of the number $M$ of fitting parameters may be replaced with the condition that the quality of the fit, i.e. the value of $\left\|K f_{M}-\Phi_{a p p r}\right\|$, is comparable with $\epsilon_{0}$.

After this work has been completed I got to know about the work [8] where an approach having some common features with the present one was considered. The techniques of Ref. [8] are different from those of the present paper. The authors consider segments of monotonicity of the solution i.e. segments of a constant sign of its derivative (and also of constant curvature of the solution). Discretizing the problem they reduce the corresponding minimum condition to the problem of the quadratic programming with linear constraints. However, no systematic way was given in Ref. [8] to find the borders of the monotonicity segments i.e. optimal positions of maxima and minima. Thus, unlike the present method, in Ref. [8] the whole problem seems to have not been solved. Besides, the issues related to the appearance of unrealistically narrow peaks discussed above (in relation to a guess as to the true number of maxima and minima) and in the last section (in relation to convergence of the method) are not considered in Ref. [8].

It may also be noted that the techniques of Ref. [8] require finding the minimum with respect to many variables (like $f_{i} \equiv f\left(E_{i}\right)$ ) with constraints. While in the present techniques finding the minimum with respect to only a few fitting parameters without constraints is required. Furthermore, the behavior of the solution when it approaches the integration limits is not reproduced exactly in the techniques of Ref. [8]. Exact in-advance-reproduction of this behavior in the present techniques increases an overall accuracy of the solution. 


\section{INTEGRAL TRANSFORM APPROACH FOR CALCULATING REACTIONS IN QUANTUM MECHANICS}

The material of the present section is used below to generate realistic inputs $\Phi_{\text {appr }}$. Some features of the ansatz of the preceding section for the solution $f(E)$ are also related to this material.

The techniques of the preceding section are designed, in partcular, for applications in the framework of the approach that is reviewed below. Only a brief outline of the approach is contained here. More details can be found in the reviews [6, 9]. The approach is advantageous, in particular, in problems with many open channels of various nature i.e. when energy is not low.

Its main features are the following. The dynamics calculations to be performed are bound-state type calculations. In the course of calculations there is no need to consider reaction channels, as well as reaction thresholds. Reaction channels and thresholds come into play at merely the kinematics level only after a dynamics calculation is done.

Continuum spectrum states never enter the game. In place of them, "response-like" quantities of the type

$$
R(E)=\sum_{n}\left\langle Q^{\prime} \mid \Psi_{n}\right\rangle\left\langle\Psi_{n} \mid Q\right\rangle \delta\left(E-E_{n}\right)+\sum d \gamma\left\langle Q^{\prime} \mid \Psi_{\gamma}\right\rangle\left\langle\Psi_{\gamma} \mid Q\right\rangle \delta\left(E-E_{\gamma}\right)
$$

are basic ingredients of the approach. Here $\Psi_{n}$ are bound states and $\Psi_{\gamma}$ are continuumspectrum states. They represent a complete set of eigenstates of the Hamiltonian $H$ of a problem. The subscript $\gamma$ denotes collectively a set of continuous and discrete variables labeling the states which is symbolized in the summation over integration notation. The normalizations $\left\langle\Psi_{n} \mid \Psi_{n^{\prime}}\right\rangle=\delta_{n, n^{\prime}}$ and $\left\langle\Psi_{\gamma} \mid \Psi_{\gamma^{\prime}}\right\rangle=\delta\left(\gamma-\gamma^{\prime}\right)$ are assumed so that

$$
\sum_{n}\left|\Psi_{n}\right\rangle\left\langle\Psi_{n}\left|+\sum d \gamma\right| \Psi_{\gamma}\right\rangle\left\langle\Psi_{\gamma}\right|=I,
$$

$I$ being the identity operator.

In the method discussed the quantities $R(E)$ of Eq. (11) are obtained not in terms of the complicated states $\Psi_{\gamma}$ entering their definition but via a bound-state type calculation. And reaction observables are expressed in terms of $R(E)$ as quadratures.

Let us first explain the latter of these points. Consider strong-interaction induced reactions. Denote $\mathcal{A} \phi_{i}(E)$ and $\mathcal{A} \phi_{f}(E)$ the antisymmetrized "channel free-motion states". 
Here the subscript $i$ refers to the initial state of a reaction, the subscript $f$ refers to final states of a reaction, $\phi_{i, f}(E)$ are products of fragment bound states and of factors describing their free motion [10], and $\mathcal{A}$ denotes the operator realizing antisymmetrization with respect to identical particles [10]. Denote $\bar{\phi}_{i}(E)=\mathcal{A}(H-E) \phi_{i}(E)$ and $\bar{\phi}_{f}(E)=\mathcal{A}(H-E) \phi_{f}(E)$. One has $\bar{\phi}_{i}=\mathcal{A} V_{i}^{\text {res }} \phi_{i}$ and $\bar{\phi}_{f}=\mathcal{A} V_{f}^{\text {res }} \phi_{f}$, where $V_{i, f}^{r e s}$ are interactions between fragments in the initial and final states. Here it will be assumed that these interactions are of a short range so that the long-range inter-fragment Coulomb interaction is disregarded. ${ }^{2}$ The $T$ matrix determining the reaction rates is [10]

$$
T_{f i}=T_{f i}^{B o r n}+\left\langle\bar{\phi}_{f}(E)\left|(H-E-i \epsilon)^{-1}\right| \bar{\phi}_{i}(E)\right\rangle,
$$

$\epsilon \rightarrow+0$. Here $T_{f i}^{B o r n}$ is the simple Born contribution,

$$
T_{f i}^{B o r n}=\left\langle\phi_{f} \mid \bar{\phi}_{i}\right\rangle=\left\langle\bar{\phi}_{f} \mid \phi_{i}\right\rangle
$$

and the main problem consists in calculating the second contribution in (13) that includes the Green function $(H-E-i \epsilon)^{-1}$. This contribution may be represented as

$$
\int d E^{\prime} R_{E}\left(E^{\prime}\right)\left(E^{\prime}-E-i \epsilon\right)^{-1}
$$

where

$$
R_{E}\left(E^{\prime}\right)=\sum_{n}\left\langle\bar{\phi}_{f}(E) \mid \Psi_{n}\right\rangle\left\langle\Psi_{n} \mid \bar{\phi}_{i}(E)\right\rangle \delta\left(E^{\prime}-E_{n}\right)+\sum d \gamma\left\langle\bar{\phi}_{f}(E) \mid \Psi_{\gamma}\right\rangle\left\langle\Psi_{\gamma} \mid \bar{\phi}_{i}(E)\right\rangle \delta\left(E^{\prime}-E_{\gamma}\right)
$$

The quantity (15) is just of Eq. (11) structure (with the $E \rightarrow E^{\prime}$ replacement). Thus, indeed, to calculate matrix elements of the $T$ matrix it is sufficient to have quantities of this structure. Once they are available the integrations (14) are readily done.

In order to calculate a perturbation-induced reaction amplitude $\left\langle\Psi_{f}^{-}|\hat{O}| \Psi_{0}\right\rangle$ where $\hat{O}$ is a perturbation and $\Psi_{0}$ is an unperturbed initial state the same is to be done with the $\bar{\phi}_{i} \rightarrow \hat{O} \Psi_{0}$ replacement in the above relations.

Now let us explain the above mentioned point on calculating the Eq. (11) type quantities. It should also be noted that such quantities may be of interest themselves representing observable response functions for inclusive perturbation-induced reactions. Let us rewrite

\footnotetext{
2 This restriction can be weakened or removed. This is done in part in [1].
} 
Eq. (11) as

$$
\begin{gathered}
R(E)=\sum_{n} R_{n} \delta\left(E-E_{n}\right)+f(E), \quad R_{n}=\left\langle Q^{\prime} \mid \Psi_{n}\right\rangle\left\langle\Psi_{n} \mid Q\right\rangle, \\
f(E)=\sum d \gamma\left\langle Q^{\prime} \mid \Psi_{\gamma}\right\rangle\left\langle\Psi_{\gamma} \mid Q\right\rangle \delta\left(E-E_{\gamma}\right) .
\end{gathered}
$$

Calculation of the bound-state contributions $R_{n}$ can be done separately, see also below. The contribution (17) includes an integral over few- or many-body continuum states $\Psi_{\gamma}$ that are very complicated except for low energies, and the problem just lies in calculating this contribution. If $E_{t h r}$ denotes the threshold value for continuum state energies then $f(E)$ is different from zero at $E_{t h r} \leq E \leq \infty$.

An easy task is the sum-rule calculation. Using Eq. (12) one gets

$$
\int_{E_{t h r}}^{\infty} f(E) d E=\left\langle Q^{\prime} \mid Q\right\rangle-\sum_{n} R_{n} .
$$

Obviously, the quantity (18) does not allow reconstruction of $f(E)$ itself. To achieve this goal, let us consider "generalized sums" of the form

$$
\int_{E_{t h r}}^{\infty} K(\sigma, E) f(E) d E .
$$

Using Eq. (12) one obtains "continuous sum rules"

$$
\begin{array}{r}
\int_{E_{t h r}}^{\infty} K(\sigma, E) f(E) d E=\sum d \gamma\left\langle Q^{\prime} \mid \Psi_{\gamma}\right\rangle K\left(\sigma, E_{\gamma}\right)\left\langle\Psi_{\gamma} \mid Q\right\rangle \\
=\left\langle Q^{\prime}|K(\sigma, H)| Q\right\rangle-\sum_{n} R_{n} K\left(\sigma, E_{n}\right)
\end{array}
$$

where as above $H$ is the Hamiltonian of the problem and $R_{n}$ are defined in Eq. (16). If one is able to calculate the quantity $\left\langle Q^{\prime}|K(\sigma, H)| Q\right\rangle$ entering Eq. 20 then one comes to the integral equation (1) for $f(E)$ with

$$
\Phi(\sigma)=\left\langle Q^{\prime}|K(\sigma, H)| Q\right\rangle-\sum_{n} R_{n} K\left(\sigma, E_{n}\right) .
$$

And at proper choices of the kernel $K$ one can completely reconstruct $f(E)$ from this equation.

The presented approach to calculate reactions has been introduced in [12]. ${ }^{3}$ The transforms with the kernels $K(\sigma, E)=(E-\sigma)^{-p}$ where $p=1$ and 2 were employed. These are

\footnotetext{
${ }^{3}$ For observable responses $R(E)$, i.e. in case of inclusive perturbation-induced reactions, a bound-state type calculation of their integral transforms has been suggested in [13] in case of the Stieltjes transform and in [14 in case of the Laplace transform. Inversions of the transforms were not considered in those works.
} 
the Stieltjes transform and the generalized Stieltjes transform. Here $\sigma$ is chosen taking real values lower than the continuum spectrum threshold and ranging outside neighborhoods of the discrete spectrum values $E_{n}$. In accordance with Eq. (21) the input in the integral equation is

$$
\Phi(\sigma)=\left\langle Q^{\prime}\left|\frac{1}{(H-\sigma)^{p}}\right| Q\right\rangle-\sum_{n} \frac{R_{n}}{\left(E_{n}-\sigma\right)^{p}} .
$$

Denoting $(H-\sigma)^{-1} Q=\tilde{\Psi}$ and $(H-\sigma)^{-1} Q^{\prime}=\tilde{\Psi}^{\prime}$ this can also be written in the two respective cases as

$$
\begin{gathered}
\Phi(\sigma)=\left\langle Q^{\prime} \mid \tilde{\Psi}\right\rangle-\sum_{n} \frac{R_{n}}{E_{n}-\sigma} \quad \text { where } \quad(H-\sigma) \tilde{\Psi}=Q, \quad \text { and } \\
\Phi(\sigma)=\left\langle\tilde{\Psi}^{\prime} \mid \tilde{\Psi}\right\rangle-\sum_{n} \frac{R_{n}}{\left(E_{n}-\sigma\right)^{2}} \quad \text { where } \quad(H-\sigma) \tilde{\Psi}^{\prime}=Q^{\prime}, \quad(H-\sigma) \tilde{\Psi}=Q .
\end{gathered}
$$

The states $\tilde{\Psi}$ and $\tilde{\Psi}^{\prime}$ are localized. Therefore the inputs $\Phi(\sigma)$ are indeed calculable with bound-state type methods.

The transform with the "Lorentz kernel" [1] was intensively used. The procedure of Eq. (2) was used for the inversion. The kernel can be written as

$$
K(\sigma, E)=\left[\left(E-\sigma^{*}\right)(E-\sigma)\right]^{-1}
$$

where $\sigma=\sigma_{R}+i \sigma_{I}$ is now complex. The quantity $\Phi(\sigma)$ obtained in this case is of Eq. (24) form with the replacement $\left(E_{n}-\sigma\right)^{-2} \rightarrow\left[\left(E_{n}-\sigma^{*}\right)\left(E_{n}-\sigma\right)\right]^{-1}$. One can also obtain the Lorentz input $\Phi(\sigma)$ from the dynamics equations that, like the Stieltjes case (23), involve only the initial-state source term $Q$. For this purpose one rewrites the above mentioned $\Phi(\sigma)$ in the form [9]

$$
\begin{array}{r}
\Phi(\sigma)=\left(2 \sigma_{I}\right)^{-1}\left\langle Q^{\prime} \mid \tilde{\psi}_{1}-\tilde{\psi}_{2}\right\rangle-\sum_{n} \frac{R_{n}}{\left(E_{n}-\sigma^{*}\right)\left(E_{n}-\sigma\right)} \\
\text { where } \tilde{\psi}_{1}=(H-\sigma)^{-1} Q, \quad \tilde{\psi}_{2}=\left(H-\sigma^{*}\right)^{-1} Q .
\end{array}
$$

Both $\tilde{\psi}_{1}$ and $\tilde{\psi}_{2}$ are calculated from the initial state of a reaction. Final states enter here via the known quantity $Q^{\prime}$ i.e. as quadratures.

In the cases 23 and 24 it is convenient to calculate $R_{n}$ as the limits of the expression $-\left(E_{n}-\sigma\right)\left\langle Q^{\prime} \mid \tilde{\Psi}(\sigma)\right\rangle$ and of the expression $-\left(E_{n}-\sigma\right)^{2}\left\langle\tilde{\Psi}^{\prime}(\sigma) \mid \tilde{\Psi}(\sigma)\right\rangle$ respectively at $\sigma$ tending to $E_{n}$. Here $\tilde{\Psi}$ and $\tilde{\Psi}^{\prime}$ are the solutions to the corresponding inhomogeneous equations. In 
the Lorentz case one can use a similar relation with both $\sigma$ and $\sigma^{*}$ tending to $E_{n}$ i.e. with $\sigma_{R} \rightarrow E_{n}$ and $\sigma_{I} \rightarrow 0$.

Choosing the kernel $K$ as that of the Laplace transform one gets

$$
\Phi(\sigma)=\left\langle Q^{\prime}\left|e^{-\sigma H}\right| Q\right\rangle-\sum_{n} R_{n} e^{-E_{n} \sigma}
$$

This quantity is known to be calculable with the help of the Green function Monte-Carlo method at least in the cases when at each total angular momentum and parity values there exists not more than one bound state. ${ }^{4}$

In the framework of no-core shell model calculations other kernels $K$ may probably also be used in the present context, see [6, 9].

\section{EXAMPLES OF INVERSION}

In this section Eq. (1) with $E_{t h r}=0$ and with

$$
f(E)=\frac{4}{\pi E_{0}} \frac{\sqrt{E / E_{0}}}{\left[\left(E / E_{0}\right)+1\right]^{4}}
$$

taken as an exact solution is considered. Let us use $E_{0}=20.7212603615$, cf. below. Approximate solutions denoted $f_{\text {appr }}$ will be obtained below. The task here is to find out accuracy with which the approximate solutions reproduce the exact one given by Eq. (27). The minimum set of fitting parameters to be used arises when $\exp [\gamma(E)]$ is disregarded in Eq. (6). In the present one-maximum case the corresponding ansatz is

$$
f^{\prime}(E)=\frac{C}{\sqrt{E}} \frac{E-E_{1}}{[(E / \bar{E})+1]^{\beta}} .
$$

The parameters $C$ and $E_{1}$ are expressed in terms of the two other parameters $\bar{E}$ and $\beta$ with the help of the relations (5) and (8). Then the two parameters, $\bar{E}$ and $\beta$, are to be fitted. Below results pertaining to the approximation (28) are presented.

It is seen that the derivative of the solution (27) lies in the class of the functions (28). Therefore if the exact input is used then the two-parameter ansatz (28) should lead to the exact solution up to numerical inaccuracies. However, this is not the case when approximate

$\overline{4}$ At a sufficient accuracy in the calculated $\left\langle Q^{\prime}\left|e^{-\sigma H}\right| Q\right\rangle$ values the quantities $R_{n}$ and $E_{n}$ can also be treated as fitting parameters along with those entering $f(E)$. 
inputs are employed. Nevertheless, it occurs that the inclusion of additional parameters representing the quantity $\gamma(E)$ does not lead to noticeable changes of the corresponding approximate solutions obtained. ${ }^{5}$

Below some kernels $K$ of interest are considered in Eq. (1). The inputs corresponding to those kernels are $\Phi=K f$ where $f$ is the solution (27). Realistic approximate inputs $\Phi_{\text {appr }}$ related to those exact $\Phi$ are generated below and with these $\Phi_{\text {appr }}$ approximate solutions $f_{\text {appr }}$ are subsequently obtained using Eq. (9).

The average relative deviation of $f_{\text {appr }}$ from the exact $f$ is chosen as the criterion of the accuracy of a solution. I.e. the quantity

$$
\chi_{\text {solution }}=\left[\frac{1}{N_{1}} \sum_{i=1}^{N_{1}}\left(\frac{f\left(E_{i}\right)-f_{\text {appr }}\left(E_{i}\right)}{f\left(E_{i}\right)}\right)^{2}\right]^{1 / 2} \simeq\langle|\Delta f / f|\rangle
$$

is adopted as such a criterion. The sum goes over a large number of $E_{i}$ values within an appropriate range of $E$. This range is taken to be $0 \leq E \leq 42$ in all the cases, cf. Fig. 1 below.

The average relative deviation of $\Phi_{\text {appr }}$ from the exact $\Phi$

$$
\chi_{\text {input }}=\left[\frac{1}{N_{2}} \sum_{i=1}^{N_{2}}\left(\frac{\Phi\left(\sigma_{i}\right)-\Phi_{\text {appr }}\left(\sigma_{i}\right)}{\Phi_{\text {appr }}\left(\sigma_{i}\right)}\right)^{2}\right]^{1 / 2} \simeq\langle|\Delta \Phi / \Phi|\rangle
$$

is taken as the criterion of the accuracy of an approximate input. The sum goes over a large number of $\sigma_{i}$ values within the ranges of $\sigma$ employed to solve the problem. These ranges are specified below. (The quantities $\Phi_{\text {appr }}(\sigma)$ have no zeros in the cases considered.)

At performing the minimization the weight function $w(\sigma)$ entering $(10)$ is chosen to be $1 /\left[\Phi_{\text {appr }}(\sigma)\right]^{2}$. I.e. the average square of the relative deviation of $K f_{\text {appr }}$ from $\Phi_{\text {appr }}$ is minimized. The corresponding fit quality is reported below as well. As the criterion of this quality the value

$$
\chi_{f i t}=\left[\frac{1}{N_{2}} \sum_{i=1}^{N_{2}}\left(\frac{\Phi_{a p p r}\left(\sigma_{i}\right)-\left(K f_{\text {appr }}\right)\left(\sigma_{i}\right)}{\Phi_{\text {appr }}\left(\sigma_{i}\right)}\right)^{2}\right]^{1 / 2} \simeq\left\langle\left|\left(\Phi_{\text {appr }}-K f_{\text {appr }}\right) / \Phi_{\text {appr }}\right|\right\rangle
$$

is taken. Here $f_{\text {appr }}$ is the approximate solution obtained via the minimization procedure of Eq. (9).

\footnotetext{
${ }^{5}$ In some cases changes in the approximate solutions due to inclusion of those parameters prove to be even less than numerical uncertainties at finding the minimum. The program "frprmn" from [7, Sec. 10.6, is used in the present calculations to search for the minima.
} 


\section{A. Lorentz transform}

The normalized Lorentz kernel (25)

$$
K\left(\sigma_{R}, E\right)=\frac{\sigma_{I} / \pi}{\left(\sigma_{R}-E\right)^{2}+\sigma_{I}^{2}}
$$

is used. The parameter $\sigma_{I}$ determines the width of the kernel. At $\sigma_{I} \rightarrow 0$ the kernel turns into $\delta\left(\sigma_{R}-E\right)$. The kernel $\tilde{K}$ of the corresponding Eq. (3) to be solved equals $-\pi^{-1} \arctan \left[\left(E-\sigma_{R}\right) / \sigma_{I}\right]$.

The corresponding input $\Phi\left(\sigma_{R}\right)$ is given in a range of $\sigma_{R}$ values. If the exact $\Phi\left(\sigma_{R}\right)$ is analytic in this range as in the applications of the preceding section then the solution corresponding to this $\Phi\left(\sigma_{R}\right)$ exists, it is unique, and it is independent of the chosen $\sigma_{R}$ range. Indeed, $f(E)$ satisfies the equation that is obtained by the replacement of the lowest integration limit $E_{t h r}$ with $-\infty$ if one sets $f(E)=0$ at $E<E_{t h r}$. Since the kernel (32) is analytic $f(E)$ thus defined satisfies the latter equation also in the whole range $-\infty<\sigma_{R}<\infty$ with $\Phi\left(\sigma_{R}\right)$ continued analytically onto this range. And the equation thus obtained is a convolution equation having a unique solution.

Results of the present inversion method are compared below with those emerging from the Eq. (2) procedure. ${ }^{6}$ The basis sets with the $E^{1 / 2}$ threshold behavior entering (2) used in almost all practical calculations performed so far are the following ${ }^{7}$

$$
\varphi_{n}(E, \alpha)=E^{n-1 / 2} e^{-\alpha E} \quad \text { and } \quad \varphi_{n}(E, \alpha)=E^{1 / 2} e^{-\alpha E / n} .
$$

Results that were obtained with these two sets are of similar quality. Below the first of the sets is employed. The corresponding calculations will be referred to as the "standard inversion".

At searching the parameters the Lorentz transforms of the approximate solutions are calculated numerically both in the standard inversion and in the present techniques cases. The same applies to inversion of the Stieltjes transforms below.

First let us present the inversion results for the case when the input $\Phi(\sigma)$ exactly corresponding to the solution (27) is employed. While this input can be calculated directly it

\footnotetext{
${ }^{6}$ In the Lorentz case the latter procedure was studied in [6, 15, 17]. In Ref. [16] some other approaches were also tested.

${ }^{7}$ More involved basis functions were used in the recent paper [18.
} 
is convenient to obtain it in another way aiming to subsequently generate $\Phi_{a p p r}(\sigma)$. Let us come to the solution (27) proceeding from the representation of Eq. (17) type. Let the states $\Psi_{\gamma}$ in $(17)$ be the eigenstates of the one-particle free-motion Hamiltonian with the orbital momentum equal to zero. In this case one may use $E_{\gamma}$ as the $\gamma$ variable in Eq. (17) and the summation sign is to be omitted. Let us also set $Q=Q^{\prime}$ in $(17)$. The quantity $f(E)$ then equals $\left|\left\langle\Psi_{E} \mid Q\right\rangle\right|^{2}$. The corresponding wave functions $\Psi_{E}(\mathbf{r})$, normalized to $\delta\left(E-E^{\prime}\right)$ as in Eq. 12, are

$$
\Psi_{E}(\mathbf{r})=\frac{c}{\sqrt{4 \pi}} \frac{\sin (k r)}{r}, \quad c=\left(\frac{2 M}{\hbar^{2} k \pi}\right)^{1 / 2} .
$$

Here $M$ is the particle mass taken below to be the mass of the nucleon, and $E=(\hbar k)^{2} /(2 M)$. Let us also set

$$
Q(\mathbf{r})=\frac{1}{\sqrt{4 \pi}} e^{-\eta r}
$$

Bound state contributions entering relations of Sec. 3 are to be omitted. This gives the expression (27) with $E_{0}=(\hbar \eta)^{2} /(2 M)$ and with the normalization factor replaced by $4 /\left(\pi E_{0} \eta^{3}\right)$. The sum rule value (8) and (18) equals $1 /\left(4 \eta^{3}\right)$. Below it is set $\eta=1 \mathrm{fm}^{-1}$. If energies are measured in $\mathrm{MeV}$ and distances in fm then one gets the $E_{0}$ value listed above.

According to the preceding section, see Eq. (24) and below, the transform $\Phi$ corresponding to Eq. (27) can be calculated as

$$
\Phi\left(\sigma_{R}\right)=\int_{0}^{\infty}|\psi(r, s)|^{2} d r
$$

where $\psi$ is determined from the equation

$$
-\frac{\hbar^{2}}{2 M} \psi^{\prime \prime}-s \psi=r e^{-\eta r}
$$

with $s=\sigma_{R}+i \sigma_{I}$. The solution sought for is localized and satisfies the condition $\psi(0, s)=0$. In all the cases the inputs $\Phi$ or $\Phi_{\text {appr }}$ will be used in the range $-2 \leq \sigma_{R} \leq 41.4$ with $\sigma_{R}$ measured in $\mathrm{MeV}$ units. (One may hope to obtain the solution $f(E)$ with a reasonable accuracy in the range $0 \leq E \leq E_{\max }$ if the input of a similar accuracy in the range $-\sigma_{I} \leq \sigma \leq E_{\max }+\sigma_{I}$ is employed, cf. also Fig. 1.)

The exact input deduced from Eqs. (36) and (37) is calculated analytically. In Table 1 the results obtained with this input via the standard inversion are listed at the $\sigma_{I}$ value equal to $10 \mathrm{MeV}$. At this $\sigma_{I}$ value the width of the kernel is not very different from that of the solution, cf. Fig. 1 below. Standard inversions were usually done at such a condition. 


\begin{tabular}{|c|c|c|}
\hline$N$ & $\chi_{\text {fit }}$ & $\chi_{\text {solution }}$ \\
\hline 5 & $8.3 \cdot 10^{-4}$ & $5.2 \cdot 10^{-2}$ \\
\hline 8 & $2.3 \cdot 10^{-5}$ & $1.4 \cdot 10^{-2}$ \\
\hline 9 & $6.2 \cdot 10^{-6}$ & $1.3 \cdot 10^{-2}$ \\
\hline 10 & $4.1 \cdot 10^{-6}$ & $2.65 \cdot 10^{-2}$ \\
\hline
\end{tabular}

TABLE I: The inversion results. The exact input. The "standard inversion", $\sigma_{I}=10 \mathrm{MeV}$.

\begin{tabular}{|c|c|c|}
\hline$\sigma_{I}, \mathrm{MeV}$ & $\chi_{\text {fit }}$ & $\chi_{\text {solution }}$ \\
\hline 2 & $1.4 \cdot 10^{-7}$ & $1.6 \cdot 10^{-8}$ \\
\hline 10 & $1.45 \cdot 10^{-7}$ & $2.1 \cdot 10^{-8}$ \\
\hline 100 & $7.5 \cdot 10^{-8}$ & $3.3 \cdot 10^{-7}$ \\
\hline
\end{tabular}

TABLE II: The inversion results. The exact input. The new method, two fitting parameters.

The results in Table 1 are presented for various choices of the number $N$ of basis functions retained in the expansion (2).

It is seen that, although the exact input is used, the accuracy of the solution obtained is limited and the best accuracy attained is at the level of one per cent. This happens despite the fact that the transforms of the basis functions (33) are calculated with high accuracy. While the quality of the fit improves monotonically as $N$ increases this is not the case as to the solution obtained. When $N$ exceeds 9 the quality of the solution deteriorates and the instability due to tiny numerical inaccuracies starts to develop.

In Table 2 the results obtained with the method of the present work in the framework of the ansatz (28) are listed. Very different choices of the width parameter $\sigma_{I}$ are considered. In all the cases the accuracy of the solution obtained is incomparable with that for the above case of the standard inversion.

Now let us pass to the results at approximate inputs $\Phi_{\text {appr }}$. Let us proceed as follows. Eq. (37) is a special case of the dynamic equation $(H-\sigma) \tilde{\Psi}=Q$ from $(23)$ and $(24)$. Usually at solving such equations expansions over basis functions are employed in few-body calculations and main inaccuracies in $\Phi_{\text {appr }}$ come from truncations of the expansions. To model this situation let us solve Eq. (37) also in the form of a similar expansion. The number of terms $N_{0}$ retained in the expansion will be chosen such that $\Phi_{\text {appr }}$ will have the accuracy 


\begin{tabular}{|c|c|c|}
\hline$N$ & $\chi_{\text {fit }}$ & $\chi_{\text {solution }}$ \\
\hline 2 & $8.0 \cdot 10^{-2}$ & 0.22 \\
\hline 3 & $2.6 \cdot 10^{-2}$ & 0.12 \\
\hline 4 & $2.6 \cdot 10^{-2}$ & 0.13 \\
\hline 5 & $1.35 \cdot 10^{-2}$ & 0.71 \\
\hline
\end{tabular}

TABLE III: The inversion results. An approximate input. The "standard inversion", $\sigma_{I}=10 \mathrm{MeV}$.

at a per cent level. Specifically, let us use the approximation

$$
\psi(r, s) \simeq \sum_{n=1}^{N_{0}} c_{n}(s) \varphi_{n}(r), \quad \varphi_{n}(r)=[n(n+1)]^{-1 / 2} b^{-3 / 2} r L_{n-1}^{2}(r / b) \exp [-r /(2 b)] .
$$

Here $b=0.3 \mathrm{fm}^{-1}$ is the length parameter, and $L_{m}^{2}(x)$ are the Laguerre polynomials. The basis functions are orthonormalized. The expansion coefficients $c_{n}$ are determined from the projection linear equations. The corresponding matrix elements entering the equations and the resulting approximate inputs (36) are calculated analytically. The same is done below in the case of the Stieltjes transform.

First let us consider again the $\sigma_{I}=10 \mathrm{MeV}$ case. Let us set $N_{0}=10$ in Eq. (38). This leads to the corresponding $\Phi_{\text {appr }}$ that has a three per cent accuracy. The accuracy is defined as in Eq. (30),

$$
\chi_{\text {input }}=3.0 \cdot 10^{-2}
$$

The results of the standard inversion are presented in Table 3. One sees that at a three per cent accuracy of the input the best accuracy of the solution is twelve per cent and thus is unsatisfactory. (One should mention at the same time that many good accuracy results were previously obtained in such type problems applying the standard inversion. Probably the accuracy of the input was considerably higher than three per cent in those calculations.) Furthermore, it occurs that the approximate solutions at $N_{\max }=3$ and $N_{\max }=4$ are very close to each other. However, they are still not close to the true solution. Thus, stability with respect to the number of basis functions retained at performing the standard inversion is not sufficient for an approximate solution to be close to the true solution. Stability with respect to variations of $\Phi_{\text {appr }}$ is also required.

Now let us pass to the result obtained with the method of the present paper in the same 
case (see Eq. (39) and above it, $\sigma_{I}=10 \mathrm{MeV}$ ). One has

$$
\chi_{\text {fit }}=2.9 \cdot 10^{-2}, \quad \chi_{\text {solution }}=1.65 \cdot 10^{-2} .
$$

The accuracy of the solution obtained is even better than the accuracy $(39)$ of the input in contrast to the standard inversion results.

Perhaps the latter results might be somewhat improved due to use of basis functions decreasing as powers of $E$ at large $E$ values. This would require inclusion more non-linear parameters in each of basis functions. Anyway, the advantage of the present method is that in its frames there is no need to worry about the choice of the regularization parameter and instability does not arise at all.

Let us also list the results obtained with the present method at other $\sigma_{I}$ values. Let us first consider the case of a very narrow kernel, $\sigma_{I}=2 \mathrm{MeV}$. In this case the decrease of $\psi(r)$ as $r$ increases is slow with many oscillations present in its range of localization. Therefore many basis functions in (38) are required to reproduce $\psi(r)$ with a reasonable accuracy. With $N_{0}=60$ in (38) one gets $\chi_{\text {input }}=2.6 \cdot 10^{-2}$. Using the corresponding $\Phi_{\text {appr }}$ one obtains with the present method

$$
\chi_{\text {fit }}=2.6 \cdot 10^{-2}, \quad \chi_{\text {solution }}=8.6 \cdot 10^{-4} .
$$

The accuracy of the solution obtained is even considerably higher than that of the input in this case.

Let us now consider the opposite case of a very broad kernel, $\sigma_{I}=100 \mathrm{MeV}$. Very few basis functions in (38) are to be retained in this case to provide a reasonable accuracy of the input. At the number $N_{0}=3$ of these basis functions one gets

$$
\chi_{\text {input }}=1.55 \cdot 10^{-3} \text {. }
$$

The standard inversion is absolutely inapplicable in this case since the instability develops even for the lowest $N$ values in (2). However, the method of the present work allows to cope with this case as well. Using the corresponding $\Phi_{\text {appr }}$ one obtains with this method

$$
\chi_{\text {fit }}=4.8 \cdot 10^{-5}, \quad \chi_{\text {solution }}=7.7 \cdot 10^{-3} .
$$

The attained accuracy of the solution is still very reasonable. 
The results for this case are displayed in Fig. 1. The dashed curve represents the exact solution and the dotted curve represents the approximate one. They are practically indistinguishable. The solid curve represents the approximate input employed at performing the inversion. It is practically a constant. However, its tiny deviations from a constant prove to allow reconstructing the solution with a very good accuracy.

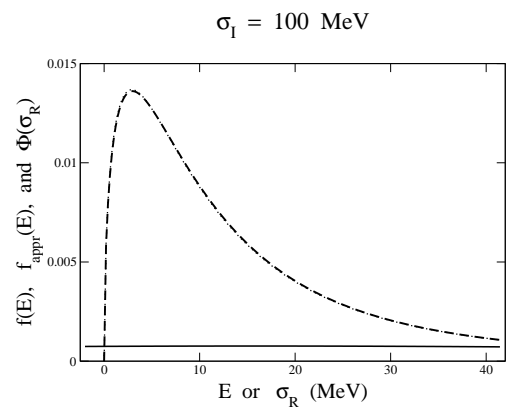

FIG. 1: The inversion results. The new method, two fitting parameters. Accuracy of the approximate input is given by Eq. (42). This input is represented by the solid curve. The dashed curve and the dotted curve represent, respectively, the exact solution and the approximate one.

If at $\sigma_{I}=100 \mathrm{MeV}$ the number $N_{0}$ of the basis functions in 38 is increased up to e.g. $N_{0}=10$ one gets

$$
\chi_{\text {input }}=9.3 \cdot 10^{-8}
$$

Using the corresponding $\Phi_{a p p r}$ one obtains in this last case with the present method

$$
\chi_{\text {fit }}=2.1 \cdot 10^{-8}, \quad \chi_{\text {solution }}=2.1 \cdot 10^{-6} .
$$

This is to be compared with the values (40) obtained at the same $N_{0}$. One sees that, at the same number of basis functions employed to solve the dynamics equation, use of a very broad kernel is more preferable in the problem than use of a kernel with the width comparable with that of the solution. This is due to faster convergence with $N_{0}$ at solving the equation (37) of the type of $(H-\sigma) \tilde{\Psi}=Q$, see the values in Eqs. 44) and 39.

Comparison between results obtained from calculations at different $\sigma_{I}$ may serve for checking purposes. 


\section{B. Stieltjes transform}

The Stieltjes kernel is

$$
K(s, E)=(E-s)^{-1}
$$

Eq. (1) is solved with $\Phi(s)$ given in a range of real negative $s$ values. If the exact $\Phi(s)$ is analytic in this range as in the applications of Sec. 3 then there exists a unique solution corresponding to this $\Phi(s)$ that is independent of this $s$ range. This follows from the fact that the Stieltjes transform is the iterated Laplace transform. Eq. (3) with the kernel $-\ln (E-s)$ is solved.

The same procedure as above that involves Eqs. (34) and (35) is used to calculate the exact input and approximate ones. As it is seen from the preceding section, Eq. (23), the exact input $\Phi$ that corresponds to the solution $(27)$ can be calculated in the Stieltjes case as

$$
\Phi(s)=\int_{0}^{\infty} \psi(r, s) r e^{-\eta r} d r
$$

where $\psi(r, s)$ is the localized solution to Eq. (37) at the condition $\psi(0, s)=0$. The same units and the same $\eta$ value as above are used. The same procedure of expanding over the basis set (38) is used to obtain approximate inputs.

At performing the inversion the inputs in the ranges $s_{\max } \geq s \geq s_{\min }$ are employed with various $s_{\max }$ and with $s_{\min }=s_{\max }-41.4 \mathrm{MeV}$. To this aim, it is expedient that $|s|$ is confined to values not too much exceeding those $E$ values at which $f(E)$ is substantially different from zero, cf. Fig. 1. At larger $|s|$ values the transform behaves as const $\cdot s^{-1}$ and it contains little additional information on $f(E)$. As to the choice of the $s_{\max }$ value, the transform exists for $s<0$ only and convergence of the approximate transform deteriorates when $s$ approaches zero.

At $s_{\max }=-2 \mathrm{MeV}$ in the case of the exact input one obtains

$$
\chi_{\text {fit }}=3.45 \cdot 10^{-12}, \quad \chi_{\text {solution }}=3.9 \cdot 10^{-11} .
$$

In the case of an approximate input obtained with $N_{0}=5$ in 38 one has $\chi_{\text {input }}=1.3 \cdot 10^{-2}$. Performing inversion with the corresponding $\Phi_{\text {appr }}$ one gets

$$
\chi_{\text {fit }}=5.5 \cdot 10^{-3}, \quad \chi_{\text {solution }}=0.10 .
$$


While this result is unsatisfactory, the increase of the number $N_{0}$ of the basis functions up to $N_{0}=7$ leads to

$$
\chi_{\text {input }}=2.2 \cdot 10^{-3}, \quad \chi_{\text {fit }}=1.4 \cdot 10^{-3}, \quad \chi_{\text {solution }}=1.5 \cdot 10^{-2} .
$$

Let us mention that for obtaining the solution with a similar accuracy when the standard inversion is used the accuracy of the input should be at the level of $10^{-5}-10^{-6}$.

If at the same $s_{\max }=-2 \mathrm{MeV}$ value one increases further the number of retained basis functions up to $N_{0}=10$ one obtains

$$
\chi_{\text {input }}=1.2 \cdot 10^{-3}, \quad \chi_{\text {fit }}=1.4 \cdot 10^{-4}, \quad \chi_{\text {solution }}=1.1 \cdot 10^{-3} .
$$

If at the same $N_{0}=10$ value one uses $s_{\max }=-10 \mathrm{MeV}$ and $s_{\max }=-20 \mathrm{MeV}$ one obtains, respectively,

$$
\begin{aligned}
& s_{\text {max }}=-10 \mathrm{MeV}, \quad \chi_{\text {input }}=1.4 \cdot 10^{-7}, \quad \chi_{\text {fit }}=9.9 \cdot 10^{-8}, \quad \chi_{\text {solution }}=2.2 \cdot 10^{-6} . \\
& s_{\text {max }}=-20 \mathrm{MeV}, \quad \chi_{\text {input }}=2.3 \cdot 10^{-9}, \quad \chi_{\text {fit }}=1.5 \cdot 10^{-9}, \quad \chi_{\text {solution }}=1.0 \cdot 10^{-7} .
\end{aligned}
$$

Thus, despite the fact that the ratio $\chi_{\text {solution }} / \chi_{\text {input }}$ becomes less favorable as $s_{\text {max }}$ decreases, the accuracy of the solution itself increases. The reason for this is similar to that in the Lorentz transform case.

Comparison of the results obtained from calculations at different $s_{\max }$ values may serve for checking purposes. Besides, use of the Lorentz and Stieltjes transforms simultaneously for the same purposes is convenient. Indeed, the dynamic equations of Eq. (37) type are the same in both cases. In addition, in the Lorentz case it is in general convenient to calculate $\Phi$ from the Stieltjes type relations, see Eq. (26).

\section{Laplace transform}

When conventional regularization methods are used to invert the Laplace transform the instability problem is known in general to be severe. The Laplace transform considered here is

$$
\int_{0}^{\infty} e^{-z E} f(E) d E=\Phi(z), \quad f(0)=f(\infty)=0 .
$$

Inversion is performed in the form

$$
\int_{0}^{\infty} e^{-z E} f^{\prime}(E) d E=z \Phi(z) .
$$


The right-hand side values in the range $0 \leq z \leq z_{\max }$ with $z_{\max } \simeq 1.9304 \mathrm{MeV}^{-1}$ are used when performing the inversion. If $\tilde{E}$ is a typical scale pertaining to the solution $f(E)$, cf. Fig. 1, then at $z \gg 1 / \tilde{E}$ the transform takes its asymptotic form and contains little additional information on $f(E)$. In the present case only minimization on grids is performed without a subsequent conventional minimization.

The transform $\Phi(z)$ of the exact $f(E)$ of Eq. (27) is calculated numerically. The inversion with this accurate $\Phi(z)$ gives (using the two-parameter ansatz (28))

$$
\chi_{\text {fit }}=2.6 \cdot 10^{-6}, \quad \chi_{\text {solution }}=8.0 \cdot 10^{-6} .
$$

In the literature, in many-body calculations inputs to Laplace transforms are obtained with the help of Monte-Carlo integrations. Therefore it will be assumed here that inaccuracies of an input are of a random nature. Random inaccuracies may be modeled in various ways. Below it is done as follows. At each $z$ value the quantity $v \Phi(z)$ is added to the accurate input $\Phi(z)$ where $v$ is a random variable such that its mean value is zero. Let us denote $\tau^{2}$ its dispersion, i.e. $\left\langle v^{2}\right\rangle$. One may set $v=\tau \varrho$ where $\varrho$ is a random variable with the unit dispersion. It is assumed that the distribution of $\varrho$ is the normal distribution and the values of $\varrho$ are taken randomly in accordance with this distribution [7] at each $z=z_{i}$ value.

The average relative error $\tau$ of the input is taken to be $5.0 \cdot 10^{-2}$. Inversion with the approximate input $\Phi_{\text {appr }}(z)$ thus generated gives

$$
\chi_{\text {fit }}=4.9 \cdot 10^{-2}, \quad \chi_{\text {solution }}=8.9 \cdot 10^{-3} .
$$

The value of $\chi_{f i t}$ is such as one could expect. The result for $\chi_{\text {solution }}$ obtained may be considered definitely good taking into account rather large relative error in the input.

\section{CONVERGENCE OF THE METHOD}

In this section it is shown that if both the quantity $\left\|\Phi-\Phi_{a p p r}\right\|$ is small and saturation is achieved with respect to the number $M$ of parameters retained e.g. in Eq. (7) then the approximate solution $f_{M}$ from Eq. (9) is necessarily close to the true $f$ everywhere except perhaps for the points of maxima and minima of $f$. The case when the uncertainty $\Phi-\Phi_{\text {appr }}$ is not random is considered here so that one can speak of the norm $\left\|\Phi-\Phi_{\text {appr }}\right\|$. (In the 
above example the method works well also in the case of random uncertainty.) Besides, the influence of round-off errors on the minimization procedure $(9)$ is disregarded below.

1. First, let us show the following. Let $f_{M}$ be the solution to Eq. (9). If $\left\|\Phi-\Phi_{\text {appr }}\right\|$ is small and saturation with respect to the number $M$ of parameters determining $f_{M}$ is achieved then the quantity $\left\|\Phi-K f_{M}\right\| \equiv\left\|K\left(f-f_{M}\right)\right\|$ is small as well. Namely,

$$
\left\|K\left(f-f_{M}\right)\right\| \leq 2\left\|\Phi-\Phi_{a p p r}\right\|+\epsilon_{1}
$$

where $\epsilon_{1}$ may be done arbitrarily small due to increase in the number $M$ of parameters retained.

Indeed, one has

$$
\left\|K\left(f-f_{M}\right)\right\| \leq\left\|\Phi-\Phi_{a p p r}\right\|+\left\|\Phi_{a p p r}-K f_{M}\right\|
$$

Let $f_{M}^{0}$ be an arbitrary function of the structure of Eqs. (6) and (7) with the same $M$ parameters retained as in the case of $f_{M}$. Due to Eq. (9) one can write

$$
\left\|\Phi_{\text {appr }}-K f_{M}\right\| \leq\left\|\Phi_{\text {appr }}-K f_{M}^{0}\right\| \leq\left\|\Phi_{\text {appr }}-\Phi\right\|+\left\|\Phi-K f_{M}^{0}\right\| .
$$

Let us choose $f_{M}^{0}$ to be arbitrarily close to exact $f$ taking $M$ value to be sufficiently large. Since the $K$ operator is continuous, at any $\epsilon_{1}>0$ one can find such $\delta$ in the corresponding relation $\left\|f-f_{M}^{0}\right\|<\delta$ that one gets $\left\|\Phi-K f_{M}^{0}\right\|<\epsilon_{1}$. Then combining Eqs. (55) and (56) one obtains Eq. (54). (The above norm in the $f$ space may be defined differently from that in the $\Phi$ space.)

2. Now the following question is to be addressed. Suppose that $\left\|K\left(f-f_{M}\right)\right\|$ is small. What are implications of this fact for the behavior of $f-f_{M}$ itself?

Below let us define the $K$ operator as follows,

$$
(K \psi)(x)=\int_{a}^{b} K(x, y) \psi(y) d y, \quad c \leq x \leq d
$$

where $K \psi \in L_{2}(c, d)$, and the functions $\psi$ belong to the class of piecewise continuous functions. The notation $\|F\|$ will refer to the $L_{2}$ norms below. Performing a change of variables the norm of Eq. (10) may be rewritten as the $L_{2}$ norm. Let us also assume that $K$ is such that if $\|K \psi\|=0$ then $\|\psi\|=0$ in the class of piecewise continuous $\psi$. (This condition is equivalent to the condition that the solution to the equation $K f=\Phi$ is unique also in the class of piecewise continuous $f$.) 
Consider the mapping $K z=u$, where $z(y)$ are smooth uniformly bounded functions, $\|z\|<Z$, where $\|z\|$ is the $L_{2}(a, b)$ norms of $z(y)$. Let $\|u\|$ be the $L_{2}(c, d)$ norm of $u(x)$.

The following will be proved. At any $y_{1}$ and $y_{2}$ belonging to $[a, b]$ and at any $\epsilon>0$ one can find such $\delta$ that the relation

$$
\left|\int_{y_{1}}^{y_{2}} z(y) d y\right|<\epsilon
$$

holds true for all $z$ satisfying the condition $\|u\|<\delta$. (Here $u=u[z]$.) One can split the $[a, b]$ integration domain from Eq. (57) into segments of a given length $\Delta=y_{2}-y_{1}$. The above mentioned $\delta$ value may be found such that Eq. (58) fulfills simultaneously for all these segments.

Eq. (58) provides a tool for establishing the local properties of the approximate solution. Consequences for our purposes of this relation will be discussed below after its derivation. The above condition that $z=f-f_{M}$ are uniformly bounded is equivalent to the condition that $f_{M}$ are uniformly bounded. If required, the latter condition may be imposed at performing the minimization procedure.

To obtain the relation 58 let us write

$$
\|u\|^{2}=\int_{a}^{b} \int_{a}^{b} z(y) \mathcal{K}\left(y, y^{\prime}\right) z\left(y^{\prime}\right) d y d y^{\prime} \quad \text { with } \quad \mathcal{K}\left(y, y^{\prime}\right)=\int_{c}^{d} K(x, y) K\left(x, y^{\prime}\right) d x,
$$

$a \leq y, y^{\prime} \leq b$. In addition it will be assumed here that $\|\mathcal{K}\|^{2} \equiv \int_{a}^{b} \int_{a}^{b}\left[\mathcal{K}\left(y, y^{\prime}\right)\right]^{2} d y d y^{\prime}$ is finite. When $a$ or $b$ is infinite this may be not the case. Then for the purpose of the present reasoning the corresponding integration limit may be replaced with a large finite number. Indeed, such a replacement does not influence physics, and the magnitude of the norm $\|\mathcal{K}\|$ will be of no relevance below.

For this purpose in the above considered cases of the Lorentz, Stieltjes and Laplace transforms one would need to replace the infinite upper integration limit with a finite $R$ value. ${ }^{8}$ It can be seen that in those cases such a replacement leads merely to the replacement of the solution $f(y)$ with $f(y) \theta(R-y)$. It can be seen that the above mentioned property $\|K \psi\|=0 \Rightarrow\|\psi\|=0, \psi$ being piecewise continuous, is valid in the case of those transforms both in their original form and after the replacement of the upper integration limit with $R$.

\footnotetext{
${ }^{8}$ Below to employ expansions over continuum spectrum eigenfunctions pertaining to these transforms could be an alternative.
} 
The kernel $\mathcal{K}$ is the Fredholm one and is symmetric. Therefore it possesses eigenfunctions and it is possible to number them [19]. Let us denote them $\phi_{n}, n=1, \ldots$, and let us denote $\mu_{n}$ the eigenvalues, $\mathcal{K} \phi_{n}=\mu_{n} \phi_{n}$. Below let us choose $\phi_{n}$ to be orthonormalized.

Note that zero cannot be an eigenvalue of $\mathcal{K}$, and $\mu_{n}>0$ at any $n$. Indeed, according to Eq. (59) $\mu_{n}=\left(\phi_{n}, \mathcal{K} \phi_{n}\right)=\left\|K \phi_{n}\right\|^{2}$, and the kernel $K$ is such that $\left\|\phi_{n}\right\| \neq 0 \Rightarrow\left\|K \phi_{n}\right\| \neq 0$.

The set of the eigenfunctions of the kernel $\mathcal{K}$ is complete in the class of piecewise continuous functions in the sense of the approximation in the $L_{2}$ norm.

Indeed, for any function $\psi$ orthogonal to all $\phi_{n}$ the equality $\mathcal{K} \psi=0$ is valid almost everywhere in $[a, b]$ [19]. Hence $(\psi, \mathcal{K} \psi)=0$ for such $\psi$ where $(\chi, \varphi)$ denotes the standard scalar product. But according to the definition (59) the relation $(\psi, \mathcal{K} \psi)=0$ yields $\|K \psi\|=0$ which was adopted above to be not possible at $\|\psi\| \neq 0$ in case of the kernels $K$ under consideration. Thus if $\psi$ is orthogonal to all $\phi_{n}$ then $\|\psi\|=0$. I.e. the set $\left\{\phi_{n}\right\}$ is closed and therefore it is complete. The set is thus infinite.

Let us pass now now to Eq. (58). Let $c_{n}$ be the Fourier coefficients in the expansion of $z$ over $\phi_{n}$,

$$
z(y) \sim \sum_{n=1}^{\infty} c_{n} \phi_{n}(y) .
$$

Let us represent the integral in Eq. (58) as

$$
\int_{y_{1}}^{y_{2}} z(y) d y=\sum_{n=1}^{\infty} c_{n} d_{n}
$$

where $d_{n}$ are the Fourier coefficients in the expansion of $\theta\left(y_{2}-y\right)-\theta\left(y_{1}-y\right)$ over $\phi_{n}(y)$. Eq. (61) follows from the completeness property of the set $\left\{\phi_{n}\right\}$. Since all $\|z\|$ do not exceed some $Z$ value one has at any $n_{0}$

$$
\left|\sum_{n=n_{0}+1}^{\infty} c_{n} d_{n}\right| \leq Z\left[\sum_{n=n_{0}+1}^{\infty} d_{n}^{2}\right]^{1 / 2} .
$$

Taking this into account along with the fact that the function $\theta\left(y_{2}-y\right)-\theta\left(y_{1}-y\right)$ has a finite norm one sees that at any $\epsilon>0$ one can find such $n_{0}$ that the relation

$$
\left|\sum_{n=n_{0}+1}^{\infty} c_{n} d_{n}\right| \leq \frac{\epsilon}{2}
$$

is valid. The estimate is uniform with respect to $z(y)$ out of the class considered. 
Furthermore, one has

$$
\left|\sum_{n=1}^{n_{0}} c_{n} d_{n}\right| \leq S_{1} \leq S_{2} \leq S_{3}
$$

where

$$
\begin{gathered}
S_{1}=\left[\sum_{n=1}^{n_{0}} c_{n}^{2}\right]^{1 / 2}\left(y_{2}-y_{1}\right)^{1 / 2}, \\
S_{2}=\frac{1}{\mu_{\min }\left(n_{0}\right)}\left[\sum_{n=1}^{n_{0}} \mu_{n}^{2} c_{n}^{2}\right]^{1 / 2}\left(y_{2}-y_{1}\right)^{1 / 2}, \\
S_{3}=\frac{\|u\|\left(y_{2}-y_{1}\right)^{1 / 2}}{\mu_{\min }\left(n_{0}\right)},
\end{gathered}
$$

and $\mu_{\min }\left(n_{0}\right)=\min \left(\mu_{1}, \mu_{2}, \ldots, \mu_{n_{0}}\right)$. Let us set

$$
\|u\|<\frac{\epsilon}{2} \frac{\mu_{\min }\left(n_{0}\right)}{\left(y_{2}-y_{1}\right)^{1 / 2}} .
$$

Combining then Eqs. (63) and (64) one obtains that the absolute value of the quantity (61) does not exceed $\epsilon$, i.e. at arbitrary $\epsilon$ one comes to Eq. (58).

If the $[a, b]$ integration domain in Eq. (57) is split into the segments of the length of $\Delta=y_{2}-y_{1}$ then at a given $\epsilon$ one can adopt the highest of the $n_{0}$ values pertaining to these segments as the common $n_{0}$. The $\|u\|$ value in the estimate (65) can be chosen the same for all the segments. Then Eq. (58) will be valid for all the segments simultaneously.

3. The property (58) shows that at small $\|K z\|$ the quantity $z$ may be not small only because of narrow peaks of high amplitude. And if the requirement is imposed that the approximate solution $f_{M}$ has the same number of maxima and minima as the true $f$ has such narrow peaks of high amplitude are forbidden everywhere except for the points of maxima and minima of $f$. Therefore $z$ is necessarily small everywhere except possibly for these points.

Let us show this in more detail. Suppose that the $[a, b]$ integration domain is split into segments of the length of $\Delta$. Below $\Delta$ is considered to be sufficiently small. Suppose that the property (58) is fulfilled for all the segments simultaneously. This imposes the following limitation on the behavior of $z(y)$. It varies within the band $|z| \leq \epsilon / \Delta$ and out of the band peaks with widths not exceeding $2 \Delta$ are only permissible. (I.e. if a segment $\left[y_{1}, y_{2}\right]$ exists at which $z(y)>\epsilon / \Delta$ or $z(y)<-\epsilon / \Delta$ for all the $y$ values belonging the segment then necessarily $y_{2}-y_{1} \leq 2 \Delta$.) Indeed, otherwise the condition (58) would be violated at some of the segments. 
Let us exclude $\pm \Delta$ vicinities of maxima and minima of $f$ from the consideration (cf. the beginning of Sec. 2 in this connection). Let us impose now the requirement that the approximate solution $f_{M}$ has the same number of maxima and minima as the true $f$ has. This requirement puts limits on the amplitudes of the above mentioned peaks possibly present beyond the band. These amplitudes cannot exceed the $\left|\bar{f}^{\prime}\right| \cdot 2 \Delta+\epsilon / \Delta$ values where $\bar{f}^{\prime}$ are average derivatives of the true solution in those segments (of the length not exceeding $2 \Delta$ ) where the peaks are located. Indeed, otherwise, due to these peaks, the quantity $f_{M}=f-z$ would include more maxima and minima than $f$ does. But in this consideration $\Delta$ may be chosen arbitrarily small from the beginning. And when $\Delta$ is given $\epsilon(\Delta)$ may be taken arbitrarily small provided that $\left\|K\left(f-f_{M}\right)\right\|$ is sufficiently small. At this condition $|z|$ becomes smaller than any prescribed value.

Thus in the whole $[a, b]$ integration domain with the points of maxima and minima of $f$ excluded the following is valid. If the mentioned requirement on the number of maxima and minima is imposed then there exist $\delta_{1}$ values such that when $\left\|K\left(f-f_{M}\right)\right\|<\delta_{1}$, cf. Eq. (54), the $\max \left|f-f_{M}\right|$ quantity is necessarily smaller than any prescribed value.

In conclusion, techniques to solve integral equations of the first kind with an approximate input are proposed. It is proved that, at the conditions and reservations listed above, the present method provides the solution stable with respect to perturbations of an input. No regularization is required at solving a problem in this way. The fact that one need not deal with a regularization parameter may in practice lead to a higher accuracy. Some inversion problems allowing comparison with an exact solution of a rather simple structure are studied numerically. They include inversions of the Lorentz, Stieltjes and Laplace transforms and involve systematic and random errors of an input. The results prove to be very satisfactory.

Acknowledgments of financial support are given to the RFBR, Grant No. 10-02-00718 and RMES, Grant No. NS-215.2012.2.

[1] V.D. Efros, W. Leidemann, and G. Orlandini, Phys. Lett. B338, 130 (1994).

[2] A.N. Tikhonov and V.Y. Arsenin, Solutions of Ill-posed Problems, Winston, 1977.

[3] A.F. Verlan and V.S. Sizikov, Integral Equations: methods, alghoritms, programs, Kiev, "Naukova dumka", 1986. 
[4] J.E. Gubernatis, M. Jarrell, R.N. Silver, and D.S. Sivia, Phys. Rev. B 44, 6011 (1991).

[5] C.W. Groetsch, J. Phys. Conf. Ser. 73, 012001 (2007).

[6] V.D. Efros, W. Leidemann, G. Orlandini, and N. Barnea, J. Phys. G: Nucl. Part. Phys. 34, R459 (2007).

[7] W.H. Press, S.A. Teukolsky, W.T. Weterling, and B.P. Flannery, Numerical Recipes, Cambridge University Press, 1997.

[8] V.A. Morozov and A.I. Grebennikov, Methods for solution of ill-posed problems. Algorythmic aspects, Moscow University Press, 2005 (Chapter 4).

[9] V.D. Efros, Phys. At. Nucl. 62, 1833 (1999); arXiv:nucl-th/9903024.

[10] M. Goldberger, K. Watson, Collision Theory, Dover publications, N.Y., 2004.

[11] S. Quaglioni, W. Leidemann, G. Orlandini, N. Barnea, and V.D. Efros, Phys. Rev. C 69, $044002(2004)$.

[12] V.D. Efros, Sov. J. Nucl. Phys 41, 949 (1985).

[13] V.D. Efros, Ukr. Fiz. Zh. 25, 907 (1980).

[14] D. Thirumalai and B.J. Berne, J. Chem. Phys. 79, 5029 (1983).

[15] V.D. Efros, W. Leidemann, and G. Orlandini, Few-Body Syst. 26, 251 (1999).

[16] D. Andreasi, W. Leidemann, C. Reiss, and M. Schwamb, Eur. Phys. J. A 24, 361 (2005).

[17] N. Barnea and E. Livertz, Few-Body Syst. 48, 11 (2010).

[18] G. Bampa, W. Leidemann, and H. Arenhövel, Phys. Rev. C 84, 034005 (2011).

[19] F.G. Tricomi, Integral Equations, Dover publications, N.Y., 1985. 